ERRATA

$\overline{\underline{ }}$

\title{
Erratum to: "Self-Assembling Catalytic Systems Based on New Amphiphile Containing Purine Fragment, Exhibiting Substrate Specificity in Hydrolysis of Phosphorus Acids Esters" [Russian Journal of General Chemistry 86 (3), 656-660 (2016)]
}

D. A. Samarkina, D. R. Gabdrakhmanov, V. E. Semenov, F. G. Valeeva, L. M. Gubaidullina, L. Ya. Zakharova*, V. S. Reznik, and A. I. Konovalov

Arbuzov Institute of Organic and Physical Chemistry, Kazan Scientific Center, Russian Academy of Sciences, ul. Akademika Arbuzova 8, Kazan, Tatarstan, 420088 Russia

*e-mail: lucia@iopc.ru

Received July 6, 2017

DOI: $10.1134 / \mathrm{S} 1070363217070350$

ACKNOWLEDGMENTS should be as follows:

This work was financially supported by the Russian Science Foundation (project no. 14-50-00014). 